\section{MÉTODO CSORA: LA BÚSQUEDA DE CONOCIMIENTO}

\author{
Ángel Fidalgo y Javier Ponce \\ Departamento de Matemática Aplicada y Métodos Informáticos. \\ Universidad Politécnica de Madrid. \\ Ríos Rosas 21. 28003 MADRID. ESPAÑA \\ Centro para el Desarrollo Tecnológico e Industrial. \\ Ministerio de Ciencia e Innovación. \\ Cid 4. 28001 MADRID. ESPAÑA \\ angel.fidalgo@upm.es,.jpm@cdti.es
}

\begin{abstract}
In this paper we offer a fast overview of the usage needs of search engines for knowledge management, as well as the basic ideas about how they work. We then present the CSORA method (Classify, Search, Organize, Relate and Adapt), a set of technical and methodological procedures that improve the efficacy of search engines, making it possible to find relevant information in environments with lots of information or specialized information. Last, we show the integration of the method into the search engines. The conclusions include a description of the advantages of using this method, verified by experiments carried out, and the lines of future work.
\end{abstract}

KEY WORDS: Knowledge spirals; labels; categories; search engines; Web 2.0; CSORA method.

\section{INTRODUCCIÓN}

El ciclo del conocimiento es una espiral continua compuesta por varias fases, entre las que cabe destacar el fomento para la creación del conocimiento, la creación, la distribución, la aplicación y la evaluación. El considerable aumento de conocimiento social, la tendencia a convertir el conocimiento propietario en abierto, las políticas europeas para el fomento de la libre circulación de conocimiento y la facilidad de acceso al conocimiento a través de internet hacen difícil la aplicación del conocimiento, ya que es muy difícil encontrarlo en ese mar de la información qué es internet.

Uno de los principales objetivos que persigue la libre circulación del conocimiento es facilitar la aplicación del mismo. Para ello se deben disponer de sistemas de organización que permitan encontrar el conocimiento más útil para satisfacer una necesidad concreta.

\section{CSORA METHOD: THE SEARCH FOR KNOWLEDGE}

RESUMEN: En este artículo se ofrece una visión rápida de las necesidades del uso de buscadores para la gestión del conocimiento y de las ideas básicas de su funcionamiento. A continuación se presenta el método CSORA (Classify, Search, Organize, Relate, Adapt), conjunto de procedimientos técnicos y metodológicos que mejoran la eficacia de los buscadores haciendo posible encontrar información relevante en entornos con mucha información o información especializada. Finalmente se muestra su integración en los motores de búsqueda y en las conclusiones se incluyen las ventajas de su uso, verificadas a través de experiencias realizadas y las líneas de investigación futuras.

PALABRAS CLAVE: Espirales de conocimiento; etiquetas; categorías; motores de búsqueda; Web 2.0; método CSORA.

Los buscadores son las herramientas más utilizadas para buscar conocimiento y aplicarlo, sin embargo las ingentes cantidades de información que encuentran hacen poco operativos sus resultados.

La organización de los recursos es clave para encontrar una determinada información. Un ejemplo se puede encontrar en las páginas web; dependiendo de cómo esté organizada la información es más o menos fácil encontrarla. En algunas páginas web es necesario conocer prácticamente la estructura interna de la entidad para encontrar una determinada información, lo que hace más fácil utilizar un buscador que la propia página web. Si la página web se organiza en función de los posibles usuarios, es más fácil encontrar la información que interese a éstos y en esta línea están actuando un mayor número de entidades

Hace una década se pensaba que las páginas web serían el medio de búsqueda y acceso más importante a 
la información y, por tanto, tener un dominio (dirección de la página web) era clave. Debido a la proliferación de páginas web y a la dificultad para encontrar una determinada información en ella, comienzan a cobrar importancia los buscadores, siendo actualmente el medio más utilizado para el acceso a la información. Hoy en día es más importante aparecer en los primeros lugares de los buscadores que el propio nombre y dirección de la página web.

Pero la mayoría de los buscadores de internet están preparados para encontrar información no conocimiento. Los principales motores de búsqueda actúan basados en programas "arañas" que capturan información de todas las páginas web que encuentran, para después contrastarla con la información especificada en la búsqueda. Esto ocasiona que, ante cualquier texto de búsqueda, aparezcan millones de referencia. En estas circunstancias es fundamental que la información buscada aparezca en los primeros lugares de la lista de referencias encontradas, en caso contrario será muy difícil encontrar la información deseada. Por tanto, encontrar información relevante en internet depende más de la experiencia en la búsqueda, las aptitudes, la inventiva y buena suerte (Gresman, 1988) más que de los propios resultados de la búsqueda, esto se debe a que no hay una fórmula matemática que permita el mejor ordenamiento (Chakrabarti, 1999).

Actualmente la investigación en motores de búsqueda se basa en programas de análisis semántico, de tal forma que lo que aparezca en los primeros lugares de la búsqueda sea información relevante; es decir, conocimiento. En cualquier caso (en los motores de búsqueda actuales y futuros), lo que se mantiene es una constante: antes de realizar la búsqueda se debe tener muy claro qué se quiere buscar.

No obstante, la irrupción de la denominada Web 2.0 en internet nos ha proporcionado otro método de búsqueda: "las etiquetas". Una de las características de la Web 2.0 es que los usuarios de la aplicación son los que suben sus propios contenidos, por tanto para los desarrolladores de la web les resulta totalmente imposible clasificar y categorizar la información a priori, antes de que esta sea subida por los usuarios. Las etiquetas nacen como un sistema para clasificar la información que los pro- pios usuarios introducen en la página web; el software web 2.0 permite buscar la información a partir de las etiquetas que se han usado para la clasificación. Hay tres métodos para buscar la información a partir de las etiquetas:

- El índice de etiquetas (Figura 1-a). La principal característica es que se puede ver una relación entre las etiquetas. La relación suele ser jerárquica y suele estar representada en forma de índice.

- La nube de etiquetas (Figura 1-b). La principal característica de este tipo de búsqueda es que se resalta la etiqueta más utilizada. Es una forma visual de organizar las etiquetas, o lo que es lo mismo la clasificación de la información a buscar.

- La lista de etiquetas (Figura 1-c). La principal característica es que se muestra una lista ordenada de etiquetas; es decir, se puede ver la clasificación empleada en la distinta información de la web en orden alfabético. Opcionalmente se permite ver el número de referencias junto a la etiqueta.

Las búsquedas por etiquetas tienen dos características comunes, independientemente del método empleado para organizar y visualizar las etiquetas:

- Únicamente se puede buscar por una etiqueta, mostrando la información relacionada con la misma. En los casos de las búsquedas por listas o nubes es una característica lógica; sin embargo en la búsqueda por índices no relaciona las etiquetas; es decir, si se toma una etiqueta de tercer nivel, no muestra la información que está en niveles anteriores. Tomando como ejemplo la Figura 1-a, si se selecciona la etiqueta "Web 2.0" nos mostraría la información que tiene esta etiqueta, no nos mostraría la información que tuviese las etiquetas "tecnología educativa" + "Web 2.0".

- El resultado de la búsqueda es una lista de referencias de información relacionadas con la etiqueta seleccionada.

En las siguientes secciones se presenta y describe el método CSORA, desarrollado y experimentado por los autores, que, a partir de procesos técnicos y metodológicos, mejora la eficacia de los buscadores de conocimiento. 


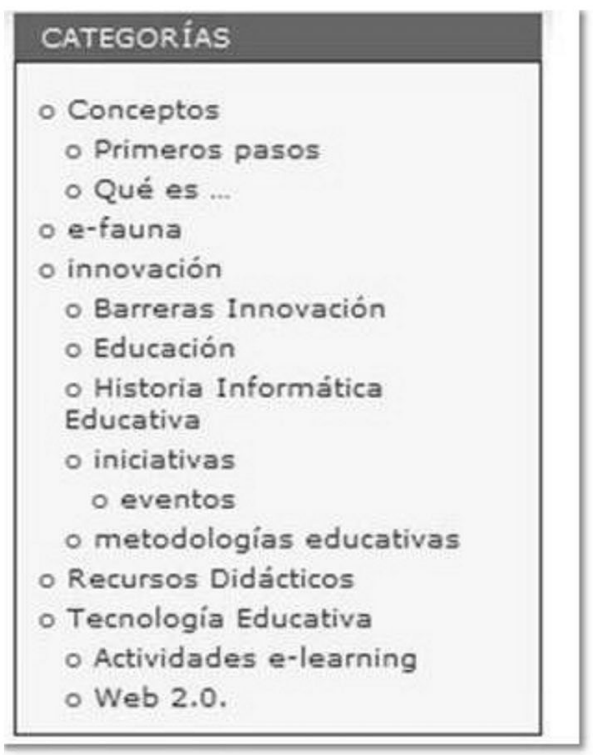

(a) ORGANIZACIÓN EN ÍNDICE. LAS ETIQUETASESTÁN RELACIONADAS DEFORMA JERÁRQUICA.

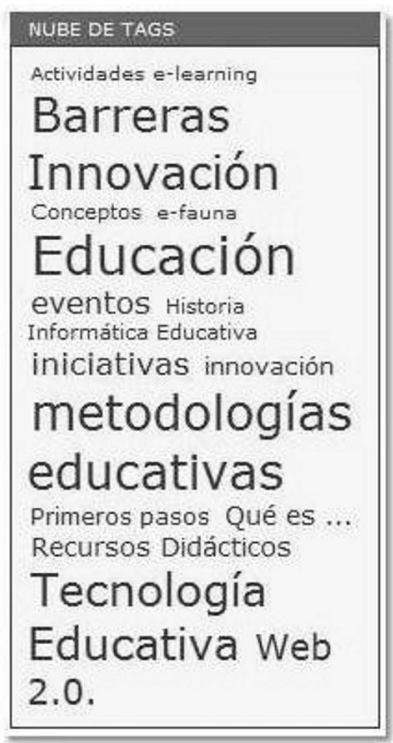

\begin{tabular}{|l|}
\hline LISTA DE EIIQUETAS \\
\hline Elegir categoria \\
\hline Elegir categoria \\
\hline Actividades e-learning (2) \\
Barreras Innovación (56) \\
Conceptos (6) \\
e-fauna (2) \\
Educación (63) \\
eventos (24) \\
Historia Informática Educativa (5) \\
iniciativas (26) \\
innovación (12) \\
metodologias educativas (58) \\
Primeros pasos (10) \\
Qué es - (17) \\
Recursos Didácticos (17) \\
Tecnología Educativa (51) \\
Web 2.0. (32) \\
\hline
\end{tabular}

(b) ORGANIZACION EN NUBE. DIFERENTETAMAÑODEETLUUETAS, CUANTOMAS GRANDEESLA ETLUETAMÁSREFERENCIAS ASOCIADASTIENE (c) ORGANIZACION EN LISTA. LISTADO ALFABETICO DELAS ETIQUETAS. OPCIONALMENTESEPUEDEPONEREL NUMMERO DEREFERENCIASASOCIADASA CADAETIQUETA

Figura 1. Diferentes tipos de organización en un sistema de búsqueda por etiquetas.

\section{Método CSORA para la búsoueda por etiquetas. Características técnicas Y METODOLÓGICAS}

Los motores de búsqueda pierden eficacia a medida que aumenta la información donde buscar o cuando se busca información en repositorios especializados en un mismo tema.

La búsqueda en contextos donde hay mucha información, por ejemplo en internet, produce resultados de búsquedas de millones de referencias ordenadas en una lista; el criterio de ordenación depende de los algoritmos de los motores de búsqueda. Esto no garantiza que las referencias que aparecen en los primeros lugares de la lista sean relevantes para la persona que ha realizado la búsqueda. Si se dedican cuatro horas a analizar los resultados de búsqueda hay más probabilidad de encontrar información relevante que si se dedica media hora, eso es evidente, por tanto la variable relacionada con la obtención de información relevante es el tiempo dedicado al análisis de los resultados de la búsqueda.

La búsqueda en repositorios especializados presenta problemas similares a la búsqueda de información en internet. El resultado suelen ser todas las referencias que hay en el repositorio. El motivo es que al ser especializado se suelen utilizar un conjunto reducido de palabras técnicas científicas o especializadas; dichas palabras suelen estar presentes en todas las referencias del repositorio.

El Laboratorio de Innovación en Tecnologías de la Información (en adelante LITI) de la Universidad Politécnica de Madrid, desarrolló un sistema de gestión de conocimiento denominado DSED (Dynamic Systems for E-Document). Dicho sistema se comenzó a utilizar como sistema de 
trabajo colaborativo (como se muestra en Sein-Echaluce, Fidalgo y Gil (2004)), lo que generaba mucha información proveniente de cientos de usuarios. El método CSORA nació como un sistema diseñado para buscar información entre todos los contenidos alli aportados.

El método CSORA (Classify, Search, Organize, Relate, Adapt) permite solventar los problemas anteriores; es decir, permite encontrar información relevante en entornos con mucha información o información especializada. Este método se basa en procedimientos tecnológicos y metodológicos para gestionar las etiquetas asociadas a la información.

Actualmente las búsquedas basadas en etiquetas tienen limitaciones técnicas y metodológicas. La principal limitación técnica es que, independientemente de la forma en que estén organizadas las etiquetas, únicamente se puede seleccionar una etiqueta para realizar la búsqueda. La principal limitación metodológica radica en que se utilizan las mismas etiquetas para clasificar la información que para buscarla.

Los procedimientos tecnológicos del método CSORA mejoran la tecnología de búsqueda existente basada en etiquetas, permitiendo más variedades de búsqueda y precisión en los resultados; esto permite encontrar conocimiento en lugar de información. Los procedimientos metodológicos permiten realizar búsquedas incluso sin saber lo que se está buscando, pero conociendo, por ejemplo, para qué se quiere utilizar.

Desde el punto de vista metodológico el método CSORA presenta una serie de características frente a los actuales sistemas de búsqueda por etiquetas:

- No utiliza las mismas etiquetas para clasificar que para buscar. Las etiquetas para la búsqueda no tienen por qué ser las mismas que para la clasificación. Este aspecto metodológico permite incorporar criterios de búsqueda distintos a los que se podrian realizar por la clasificación.

- Utiliza etiquetas para organizar los resultados de la búsqueda y para relacionar las referencias encontradas con otras.

- Permite una máxima adaptabilidad ya que se puede definir la estructura de la búsqueda, organización de resultados y forma de relacionar cada referencia.
Desde el punto de vista tecnológico el método CSORA presenta una serie de características frente a los actuales sistemas de búsqueda por etiquetas.

- Se pueden realizar búsquedas combinando las etiquetas a través de expresiones lógicas.

- Se puede elegir la organización de los resultados de las búsquedas en función de los perfiles de usuarios, o de la aplicación del conocimiento.

- Una vez localizada una referencia se pueden obtener referencias relacionadas organizadas en función de categorias.

A continuación, y para ilustrar el método CSORA, se emplearán el mismo repositorio de información junto con buscadores basados en los dos métodos de búsqueda por etiquetas, uno clásico (prototipo $\mathrm{A}$ ) y otro con el método CSORA (prototipo B).

- El prototipo A es un blog, su dirección web es http:// innovacioneducativa.wordpress.com/, donde se han implementado la organización de etiquetas tanto por nube, indices y listas.

- El prototipo $B$ contiene la misma información que $A$, pero se ha utilizado el método CSORA de organización de etiquetas. http://www.gestionaconocimiento.com/

Los cuatro pasos descritos a continuación se fundamentan en la propia denominación del método CSORA: Clasificar, Buscar, Organizar, Relacionar y Adaptar.

\section{Paso 1. La Clasificación}

Tanto en los sistemas Web 2.0 como en los sistemas basados en el método CSORA se utiliza $\mathrm{n}$ un conjunto de etiquetas para clasificar el conocimiento. En la mayoría de los casos las etiquetas se van especificando a medida que se añade la información; estas etiquetas se suelen organizar en forma de lista y cuando se tiene un número elevado de ellas se organizan como un índice. La mayoría de las aplicaciones 2.0 no disponían inicialmente de la posibilidad de organizar las etiquetas por índices por lo que es muy habitual que las etiquetas estén organizadas en listas 0 en nubes.

La forma técnica de asignar las etiquetas en los dos prototipos es muy similar (Figura 2). 


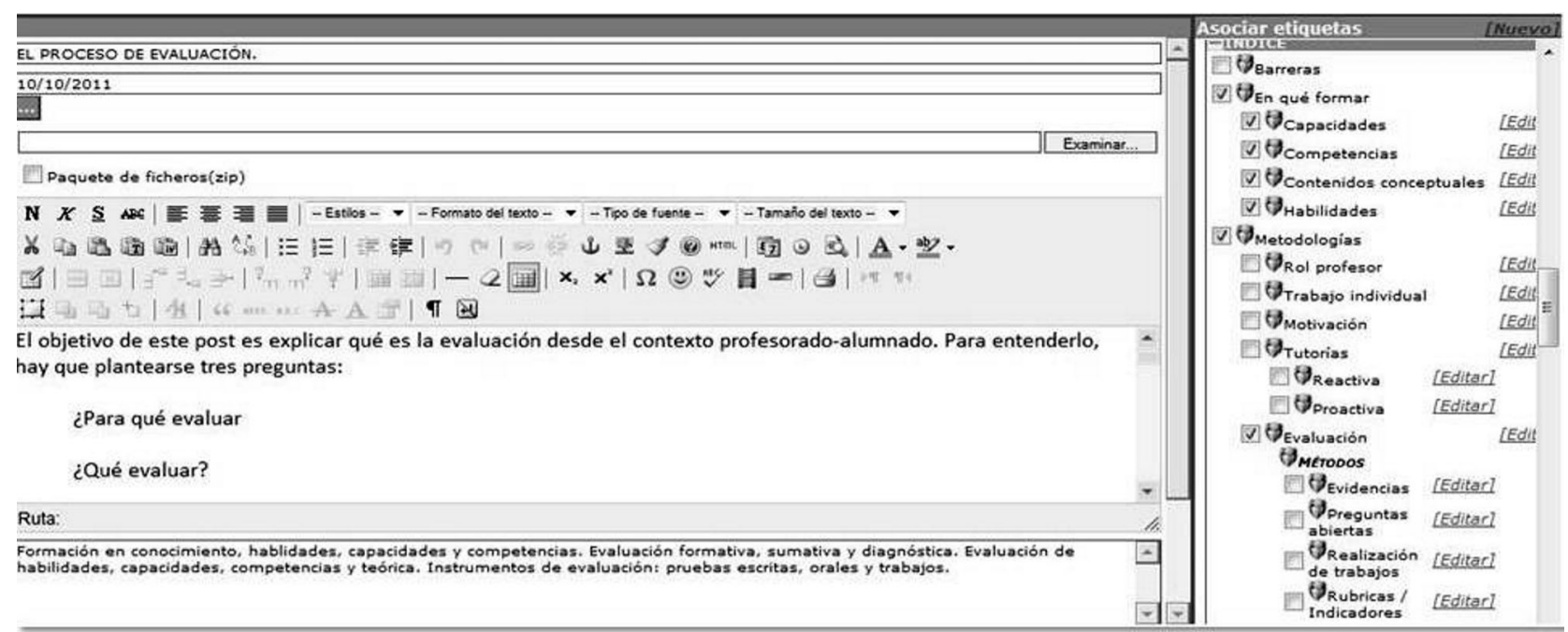

ASIGNACIÓN DE ETIQUETAS A UNA INFORMACIÓN. LA FORMA DE RELACIONAR ETIQUETAS A UNA DETERMINADA INFORMACIÓN ES COMÚN EN AMBOS MÉTODOS, SE SUELE PRESENTAR UNA INFORMACÓN ESTRUCTURADA EN UN SERIE DE CAMPOS (Titulo, Fecha, DESCripción, ...) Y CONJUNTO DE ETIQUETAS UTILIZADAS PARA CLASIFICAR LA INFORMACIÓN DEL REPOSITORIO. BASTA CON MARCAR LAS ETIQUETAS RELACIONADAS CON LAINFORMACIÓN.

Figura 2. Método clásico de asignación de etiquetas a una información.

Las principales diferencias entre ambos métodos son metodológicas. El método CSORA permite crear un completo índice de etiquetas para clasificar cualquier contenido, presente y futuro. El método clásico solo permite un índice de etiquetas del contenido actual, no del futuro.

En el método del prototipo A (método clásico) el índice de etiquetas se crea a medida que se va introduciendo la información; los índices suelen ser pequeños y con pocas etiquetas, ya que las únicas etiquetas existentes son las que posee la información contenida previamente en el repositorio. Este método es lógico y necesario ya que si se incluyen etiquetas que no están asociadas a la información del repositorio, entonces en la búsqueda aparecerán etiquetas que no tienen asociado ningún contenido y no darán ningún resultado al hacer la búsqueda a través de ellas.

En el método del prototipo $\mathrm{B}$ (método CSORA) se pueden poner cualquier etiqueta, aunque no haya información asignada a la misma. En el método CSORA las etiquetas de búsquedas no tienen por qué ser las mismas que las creadas para la clasificación de la información, por tanto no existen las limitaciones del método habitual. Sencillamente las etiquetas de la clasificación que no tienen relacionada ninguna información, no se utilizan como etiquetas de búsqueda.

La gran ventaja del método CSORA es que, en el caso de repositorios especializados, se pueden utilizar índices de etiquetas de clasificación muy completos y, si estos están estandarizados, se producirá una homogeneización del conocimiento muy importante. La Figura 3 muestra un índice de etiquetas utilizadas para la clasificación del prototipo A (limitadas por las búsquedas) y un índice en el prototipo $B$, sin ninguna limitación para clasificar.

\section{Paso 2. Las búsquedas}

Desde el punto de vista metodológico lo más importante es que el método CSORA no está obligado a utilizar las mismas etiquetas para clasificar que para buscar.

Desde el punto de vista tecnológico del método CSORA permite hacer búsquedas combinando varias etiquetas; es

ARBOR Vol. 187 Extra 3 diciembre [2011] 51-66 ISSN: 0210-1963 
decir, se pueden crear expresiones lógicas utilizando los conectores and. y .or. pero sin que el usuario necesite ningún conocimiento técnico sobre ellas ya que el procedimiento de selección es sencillo.

\begin{tabular}{l}
\hline CATEGORIAS \\
- Conceptos \\
o Primeros pasos \\
O Qué es ... \\
- e-fauna \\
- innovación \\
- Barreras Innovación \\
- Educación \\
- Historia Informática \\
Educativa \\
- iniciativas \\
o eventos \\
- metodologias educativas \\
Recursos Didácticos \\
Tecnologia Educativa \\
- Actividades e-learning \\
O Web 2.0 .
\end{tabular}

(a) INDICE. EL DETALLE EN LA ELABORACIÓN DEL INNDICEESTÁ LIMITADOPOR LA INFORMACION QUE CONTENGAELREPOSITORIO. AL SER LAS ETIQUETAS DE CLASIFICACIÓN IGUALES A LAS DE BÚSQUEDA, NO SEPUEDEN PONER ETIQUETASA LAS QUENO HAYA ASIGNADA INFORMACIÓN, YA QUELA BUSQUEDA PORLASMISMASNO MOSTRARIAN RESULTADOS.

rías. Esto no es nuevo, son varios los sistemas Web 2.0 que utilizan categorias y etiquetas, aunque no existe una aceptación común del significado y utilidad de las mismas, llegando en algunos casos a ser lo mismo una etiqueta que una categoría. Así pues, en nuestro método una categoría no es más que un nombre que aglutina un conjunto de etiquetas con unas características comunes; su misión es organizar las etiquetas en tantas categorías como se desee. Una misma etiqueta puede pertenecer a varias categorías. Las búsquedas siempre se hacen a través de etiquetas, nunca con categorias. Así mismo las

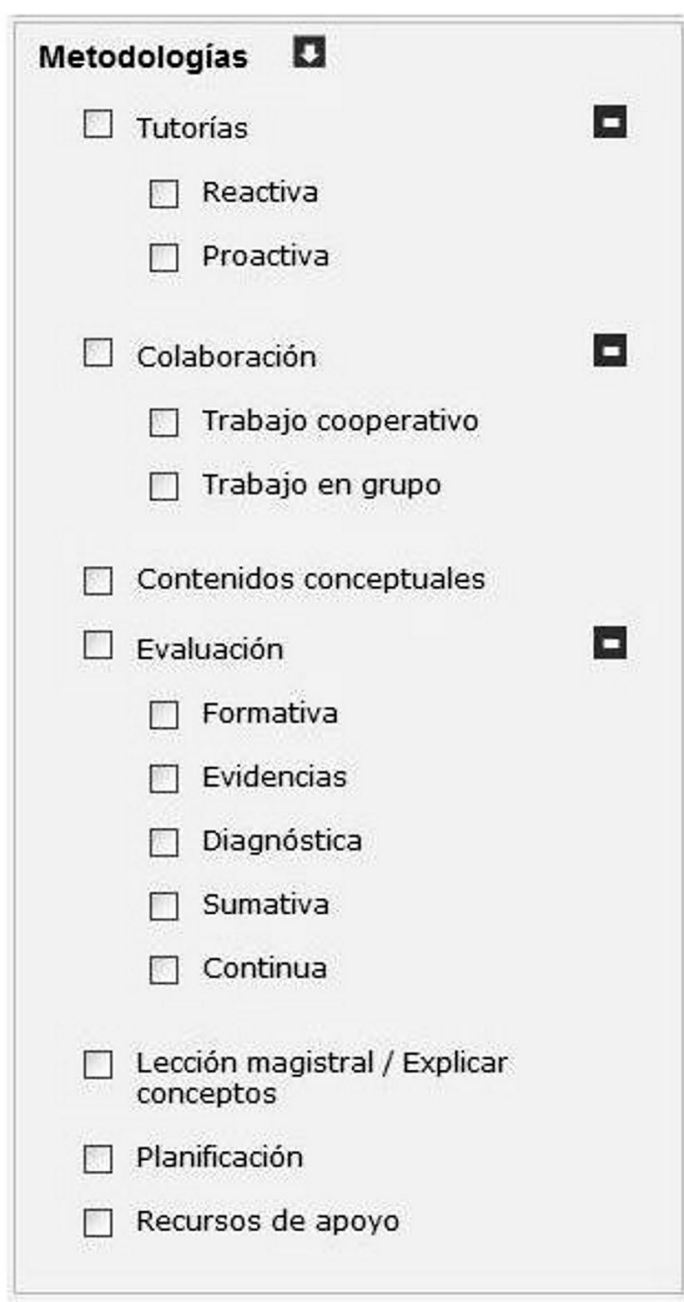

(b) INDICE CSORA. PERMITE GRAN DETALLE EN LAELABORACIÓN DELOS ÍNDICESDE ETIQUETASPARA LA CLASIFICACIÓN.

Figura 3. Detalle en la elaboración de índices de etiquetas para clasificar la información: (a) método clásico (b) método CSORA. 


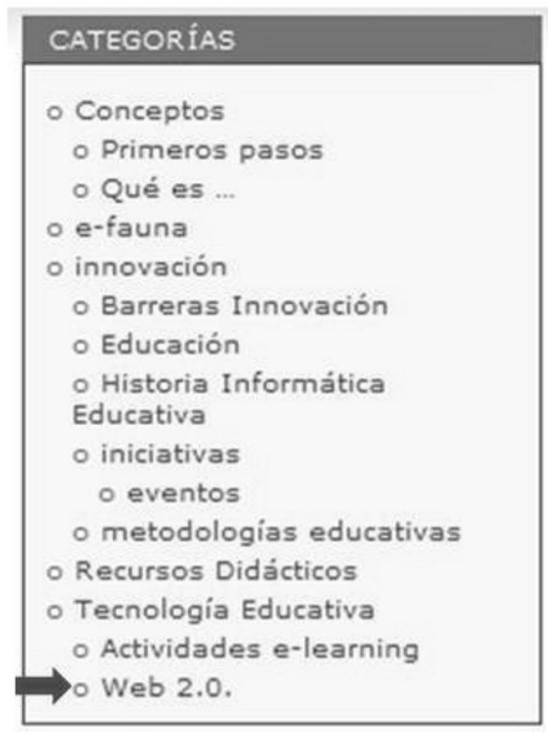

(a) BUSQUEDACLÁSICA. SE PERMITEUNA SOLAETIQUETA DE LAS QUESE HAN UTILIZADO PARA CLASIFICARLA INFORMACIÓN. HABITUALMENTETODAS LASETIQUETAS SE AGRUPAN EN UNA MISMA CATEGORÍA. EN ELEIEMPLO: BUSCARINFORMACIÓN QUECUMPLA: WEB 2.0

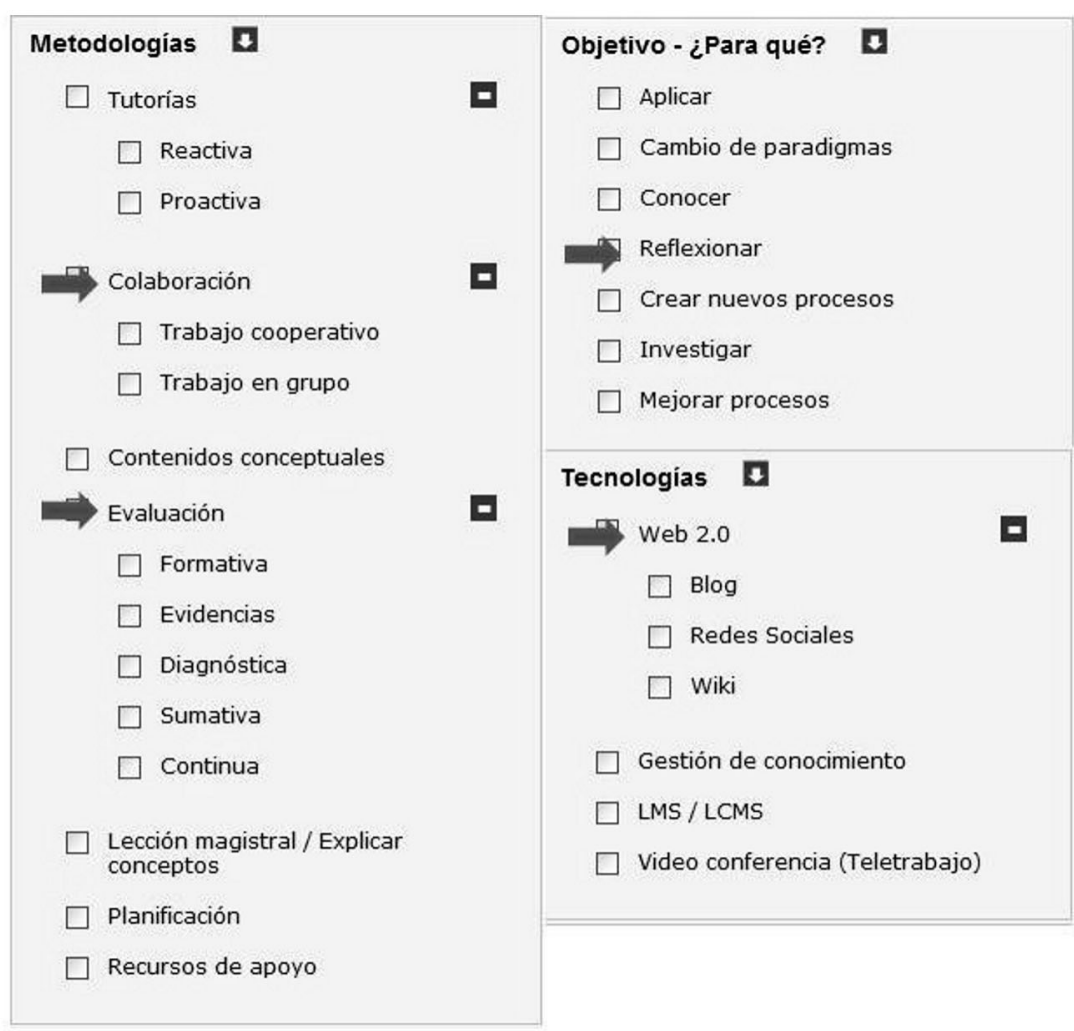

(b) BÚSQUEDA CSORA. SE PERMITE SELECCIONAR MÁS DE UNA CATEG ORIÁ Y ETIQUETA. EN EL EEEMPLO: BUSCARINFORMACIÓN QUECUMPLA:(OBJETIVO:REFLEXIONAR.Y.

TECNOLOGÍAS:WEB2.0 Y. METODOLOGÍAS (COLABORACIÓN O. EVALUACIÓN))

Figura 4. Método de búsqueda de información a través de etiquetas: (a) clásico (b) CSORA.

etiquetas de una categoría se pueden organizar como listas o como índices.

En la Figura 4 se puede ver la organización de categorias y etiquetas utilizadas para el prototipo $\mathrm{A}$ (clásico) y $\mathrm{B}$ (CSORA).

Las búsquedas para cada prototipo son las siguientes:

- En el prototipo A (Figura 4-a) solo se puede seleccionar una etiqueta. Los resultados de la búsqueda son todas aquellas referencias asociadas a la etiqueta seleccionada. En el ejemplo, todas las referencias etiquetas con la etiqueta "Web 2.0".

- En el prototipo B (Figura 4-b) se pueden seleccionar varias etiquetas. Las etiquetas de una misma categoría se tratan como un.or. y las etiquetas de distintas categorias como un.and. Se encontrarían todas las referencias sobre "Tecnologias: Web 2.0.Y. Metodologías:(Evaluación. 0. Colaboración).Y. Objetivo: Reflexionar"; es decir: el usuario requiere que el sistema muestre todas las referencias sobre web 2.0 que se utilicen en metodologías de evaluación o de colaboración y quiere esta información para reflexionar".

Desde el punto técnico, las ventajas son evidentes. Con el método CSORA se puede conseguir una precisión de búsqueda mayor que con el método del prototipo $A$ ya que se permite utilizar más de una etiqueta para las búsquedas y combinarlas en expresiones lógicas.

La tecnología del método CSORA ofrece, además, una gran ventaja metodológica, ya que se puede buscar información

ARBOR Vol. 187 Extra 3 diciembre [2011] 51-66 ISSN: 0210-1963 
sin saber qué contenido se quiere buscar. Si se utilizase la búsqueda por texto tendríamos que poner alguna palabra asociada a la información a buscar; en el método CSORA se incluyen categorias referentes, por ejemplo, a para qué utilizar la información (para reflexionar), en que procesos se va a utilizar la información (en la evaluación o en la colaboración) y exactamente qué tecnología utilizar (Web 2.0).

El método CSORA recomienda hacer un estudio previo sobre las categorías de búsquedas; si estas se organizan bien, podremos buscar información sin saber qué queremos, pero podemos saber dónde utilizarla, para qué, por qué, por quién, ... Este tipo de búsquedas son muy adecuadas para personas que no son expertas en la información a buscar, o cuando esta es muy variada o se cambia a menudo. Para conseguir ésto, nuestro método requiere que la organización de las categorías y etiquetas de búsqueda se realice teniendo en cuenta la información tanto del repositorio, como del contexto de utilización de esa información y de los perfiles de los distintos usuarios. Si se tiene en cuenta lo citado, se conseguirá un método de búsqueda que encontrará siempre información relevante; es decir, el conocimiento.

\section{Paso 3. La organización de los resultados de la búsqueda}

En relación a la forma de presentar los resultados de las búsquedas, clasificamos los buscadores en los siguientes tres tipos: "araña", "directorio" y "mixto".

1. Los buscadores de tipo "araña" (como Google) presentan los resultados de las búsquedas como una gran lista (con millones de referencias) que se organizan a través de páginas, cada página suele tener un conjunto de referencias. En la Figura 5 se muestra un resultado de búsqueda de un buscador de este La forma de organizar los resultados de búsqueda es muy adecuado para encontrar contenidos de páginas web.

2. Los buscadores de tipo "directorio" agrupan los resultados de búsqueda por categorías o índices. El resultado se muestra como un conjunto de directorios, y en cada directorio un conjunto de referencias. Las categorias suelen estar definidas por el propio buscador y las páginas previamente asociadas a esas categorias. Estos buscadores son muy adecuados para buscar páginas web clasificadas (por ejemplo, portales sobre deportes). La Figura 6 muestra un buscador que organiza el resultado de búsqueda por directorios. Desde el punto de vista organizacional la ventaja de este método es que presenta una lista ordenada de información (otra cosa es que sea o no relevante) y como principal desventaja, que la lista es demasiado grande para analizarla.

3. Buscadores "mixtos". Visualizan listas de búsqueda y directorios. Por un lado agrupan páginas web institucionales relacionadas con la palabra de búsqueda y referencias encontradas. Desde el punto de vista organizacional la ventaja es que los resultados de la búsqueda están más organizados y es más fácil acceder a un determinado tipo de información, y como principal desventaja es que no presenta la información relevante sino páginas web institucionales.

El método CSORA es un ejemplo de buscador "mixto" e integra los dos tipos de organización de los resultados de las búsquedas; es decir, permite utilizar las ventajas de ambos métodos en uno solo. Permite organizar los resultados por listas de información relevante y estas lista se organizan en categorias. En la práctica este sistema presenta varias listas y cada lista está ordenada por relevancia de la información.

Además, nuestro método permite definir una categoría sobre la que clasificar los resultados de la búsqueda. La Figura 7-a muestra una categoría para organizar el resultado de la búsqueda (con su conjunto de etiquetas) y la Figura 7-b muestra un resultado de búsqueda. Estas etiquetas se denominan etiquetas de organización y la categoría que las agrupa recibe el nombre de categoría de organización. Las etiquetas de organización pueden pertenecer también a las de clasificación y búsqueda. Como se observa en la Figura 7, en lugar de una sola lista paginada se tendrán $n$ listas, cada lista (o sub-lista) se corresponde con una etiqueta definida en una categoría denominada de organización, en la Figura esta categoría se denomina "Temática Blog Innovación Educativa". El ejemplo corresponde a la búsqueda "Innovación Educativa".

La innovación tecnológica del método CSORA se basa en la utilización de etiquetas para organizar listas; cada lista se compone de las referencias asociadas a la etiqueta, y 
Todo

Imágenes

Maps

Videos

Noticias

Shopping

Libros

Blogs

Más

Madrid

Cambiar ubicación

La Web

Páginas en español

Páginas de España

Páninas aytranioras
Innovación Educativa

innovacioneducativa.wordpress.com/

En muchas ocasiones (pero en muchas), al igual que ocurre en innovación

educativa, se han quedado con la mitad de la frase; es decir, muchas personas ...

Metodologias Educativas - + Conceptos - Un ejemplo de aprendizaje servicio.

\section{¿Qué es innovación educativa? «Innovación Educativa}

innovacioneducativa.wordpress. com/... I ique-es-innovacion-educativ...

9 Ene 2007 - Innovación Educativa en 1547. Fuente flickrCCñ Autor Ard Hesselink

Cualquier innovación introduce novedades que provocan cambios; esos ...

\section{Revista Innovación Educativa}

www. usc.es/ didoe/doc/revistalframes.htm

Review: Innovaci-n Educativa.

\section{Innovacion Educativa}

www. innovacioneducativa.net/

Jornadas de Innovación Educativa. III Encuentro Internacional de Experiencias en grupo en la Docencia Universitaria. Campus Central UCM. 27-28 de ...

\author{
[POF] Concepto INNOVACIÓN EDUCATIVA / INVESTIGACIÓN-AC... \\ www. recursoseees. uji.es/fichas/fic8.pdf \\ Formato de archivo: PDF/Adobe Acrobat - Vista rápida

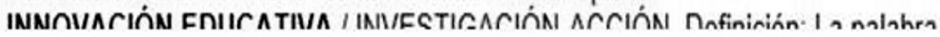

RESULTADO DE BÚSQUEDA ORGANIZADO COMO UNA GRAN LISTA. GOOGLEES EL BUSCADOR MÁPOPULARDONDELOSRESTULTADOS DELAS BÚSQUEDAS SE ORGANIZAN COMOUNA GRAN LISTA DEMILLONES DEREFEREN CIAS. PARA FACILITAR LANAVEGACIÓN LA LISTA SEPRESENTA PAGINADA. Buscadorutilizado:http://www.google.com/

Figura 5. Organización del resultado de la búsqueda en listas de referencias. Resultado obtenido para "Innovación Educativa".

en cada lista se ordenan las referencias por relevancia. Una misma información puede estar asociada a varias etiquetas; por tanto una misma referencia puede salir en varias listas.
La innovación metodológica del método CSORA, ejemplo de buscador "mixto", integra las ventajas de los buscadores que presentan los resultados en listas y de los buscadores que presentan los resultados de búsquedas en directorios. 


\section{Search innovación educativa}

\section{Open Directory Categories (1-25 of 83)}

1. World: Español: Regional: Europa: España: Gobierno: Poder Ejecutivo: Ninisterio de Ciencia e Innovacion: Consejo Superior de Deportes

2. World: Español: Regional: Europa: España: Gobierno: Poder Ejecutivo: Ministerio de Ciencia e Innovación

3. World: Español: Ciencia r tecnologia: Tecnología: Invención e innovación

4. World: Català: Ensenyament: Associacions

5. World: Português: Sociedade: Sexualidade

6. World: Español: Regional: América: Argentina: Gobierno: Poder Fjecutivo: Ninisterios: Ministerio de Ciencia, Tecnologia e Innovación Productiva: Consejo . y Técnicas

7. World: Español: Ciencia y tecnologia: Quimica: Software

8. World: Español: Regional: Europa: España: Educaciön: Organizaciones

9. World: Español: Regional: América: Argentina: Educación: Organizaciones

10. World: Español: Regional: América: Argentina: Provincias: Tucumán: Educación

11. World: Español: Regional: América: Perú: Departamentos: Arequipa: Arequipa: Educación

12. World: Español: Referencia: Museos: Infantil y juvenil

13. World: Español: Regional: América: Perú: Departamentos: Lima: Lima: Educaciōn

14. World: Español: Regional: América: Argentina: Gobierno: Poder Ejecutivo: Ninisterios: Ministerio de Ciencia. Tecnologia e Innovación Productiva

15. World: Español: Regional: Europa: España: Gobierno: Poder Ejecutivo: Ministerio de Ciencia e Innovacion: Consejo Superior de Investigaciones Cientificas

RESULTADO DE BÚSQUEDA ORGANIZADA POR DIRECTORIOS. DMOZES UNO DE LOS BUSCADORES MÁSPOPULARES QUEORGANIZA LOSRESULTADOS DE BÚSQUEDA COMO DIRECTORIOS. LOS DIRECTORIOS SUELEN AGRUPAR WEB INSTITUCIONALES FRENTEA LAS LISTAS QUEREFERENCIAN PÁGINAS YTEXTOS CONCRETOS. Buscadorutilizado:http://www.dmoz.org/

Figura 6. Organización del resultado de la búsqueda en directorios. Resultado obtenido para "Innovación Educativa".

Lo que se obtiene es una mayor precisión de búsqueda y mejor organización de los resultados. Y si la categoría de organización se realiza en función del contexto o del perfil de usuarios la eficacia de la búsqueda aumenta considerablemente.
Paso 4. Las Relaciones en la información

Desde la aparición de la Web 2.0, los medios de comunicación tradicionales (Prensa, Televisión y Radio) se han adaptado con celeridad a ella. Las noticias incorporan dos 
Los recursos encontrados con dichos criterios se agrupan de siguiente forma:

33 Contenido Web con Metodologías como Temática Blog Innovación Educativa.

24 Contenido Web con Barreras como Temática Blog Innovación Educativa.

27 Contenido Web con Tecnologías como Temática Blog Innovación Educativa.

6 Contenido Web con Web social (2.0) como Temática Blog Innovación Educativa.

12 Contenido Web con Políticas como Temática Blog Innovación Educativa.

14 Contenido Web con Historia como Temática Blog Innovación Educativa.

11 Contenido Web con Eventos como Temática Blog Innovación Educativa.

Si desea reordenar las listas por otro criterio, seleccione la opción que más se ajuste a sus necesidades de las listas desplegables y pulse reordenar

Las listas están actualmente ordenadas por Titulo - de forma Ascendente

Metodologias - Página 1 -

1 iMohicanos! a quitar piedras

Fecha: $22 / 01 / 2008$

2 ¿Cómo saber si hago innovación educativa?

Fecha: 08/04/2007

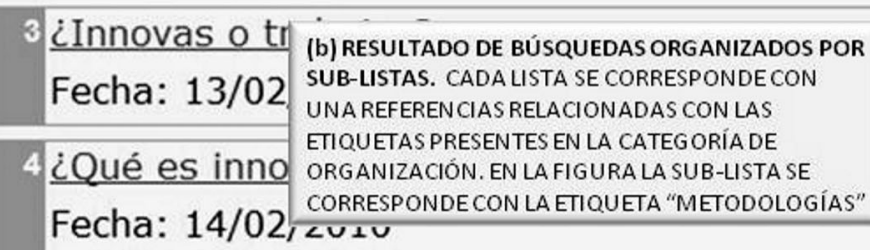

5 ¿Qué es innovación educativa?

Fecha: 09/01/2007

6 ¿Quién es el culpable de no aplicar la innovación educa

Fecha: 09/01/2009

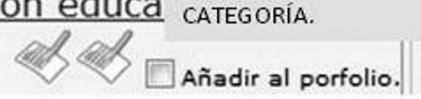

(a) ETIQUETAS DE ORGANIZACIÓN. CSORA PERMITEDEFINIR UNA CATEGORIA PARA

ORGANIZARLOSRESULTADOSDELABÚSQUEDA

A TRAVÉS DE LAS ETIQUETAS DEFINIDASEN ESA

Añadir al porfolio.

Figura 7. Organización de los resultados de búsqueda mediante el método CSORA.

características técnicas que únicamente se pueden realizar en la Web 2.0: la posibilidad de que los usuarios añadan información y la identificación de noticias relacionadas con la misma.

Los buscadores también están incorporando información relacionada con los resultados de búsquedas, la principal diferencia con las noticias 2.0 es que la relación se hace sobre las condiciones de búsqueda y se muestran organizadas en categorias, mientras que la noticias lo hacen sobre una información concreta. La Figura 8 muestra esta diferencia.
El método CSORA integra ambas características, ya que es capaz de buscar información relacionada con una referencia concreta y mostrar esta información organizada en categorías. Obsérvese que la principal diferencia con los motores de búsqueda clásicos radica en que es capaz de relacionar la información para cada referencia, no para las condiciones de búsqueda. Las Figuras 9 y 10 muestran cómo se relaciona la información para distintas referencias de búsqueda.

En el método CSORA se puede definir un conjunto de etiquetas denominadas de relación (que pueden coincidir

ARBOR Vol. 187 Extra 3 diciembre [2011] 51-66 ISSN: 0210-1963 
con las de clasificación, búsqueda y organización); estas etiquetas se agrupan en una categoría denominada de relación.

La principal ventaja tecnológica consiste en que el sistema de búsqueda es capaz de generar listas, agrupadas en categorías, para cada referencia encontrada en el resultado de la búsqueda. Los buscadores clásicos lo hacen sobre el total de la lista obtenida como resultado de la búsqueda, no con cada referencia de esa lista.

Desde el punto de vista metodológico, relacionar información organizada en etiquetas permite mostrar conocimiento relacionado con el conocimiento encontrado. Dependiendo de las etiquetas elegidas se puede relacionar la información, para cada referencia encontrada, con otra orientada a distintas aplicaciones, enfoques, procesos etc.

\section{LA INTEGRACIÓN DE LOS MÉTODOS DE BÚSQUEDAS POR ETIQUETAS CON LOS MOTORES DE BÚSQUEDAS POR INTERNET}

Actualmente la búsqueda de información suele estar basada o bien en la búsqueda por etiquetas o bien en la búsqueda por textos. Las aplicaciones Web 2.0 han incorporado la búsqueda por textos; así es fácil encontrar, en

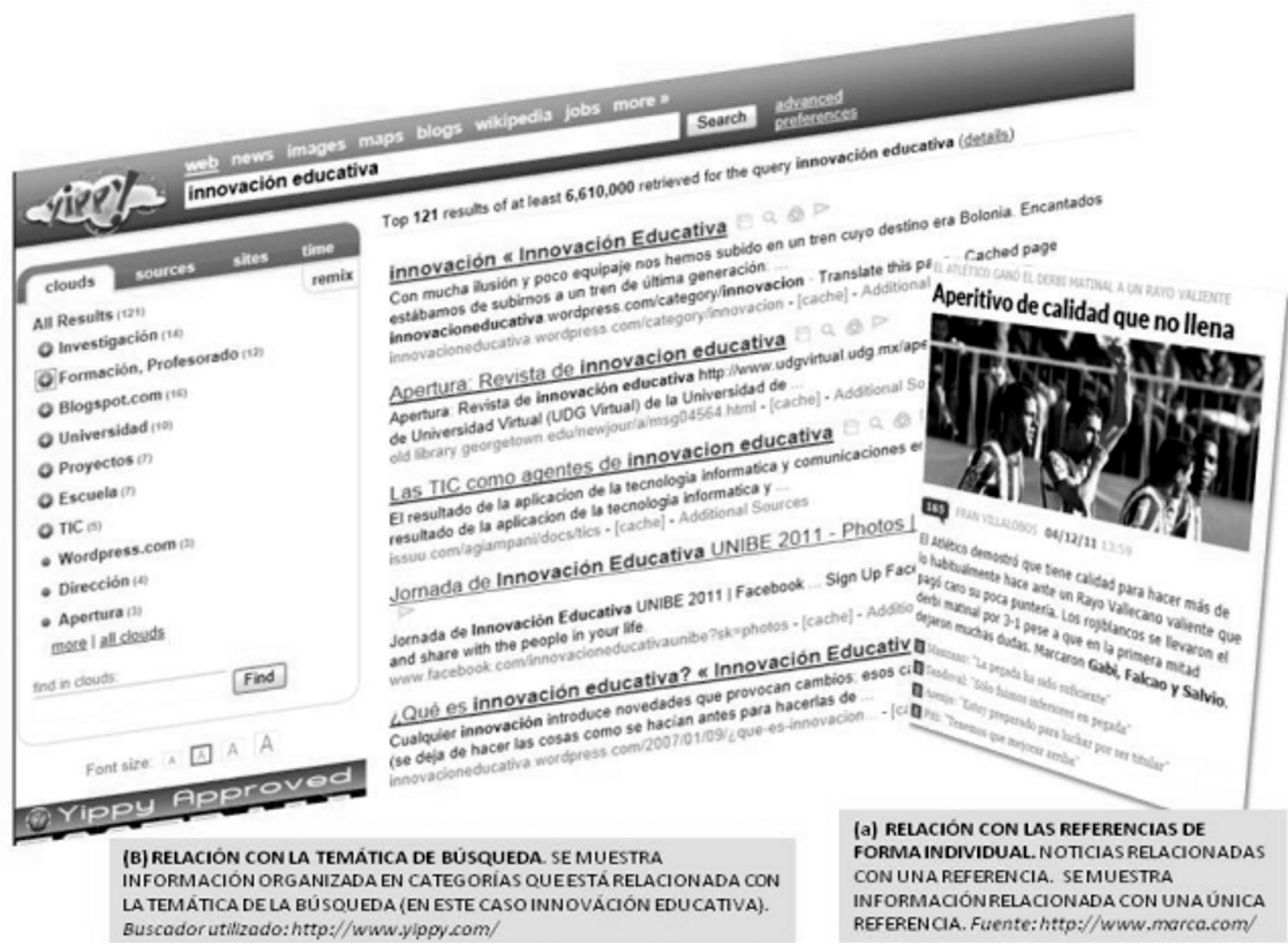

Figura 8. Relación de referencias con una única información (a) y relación de referencias agrupadas en categorías con la lista de resultados obtenidos (b). 

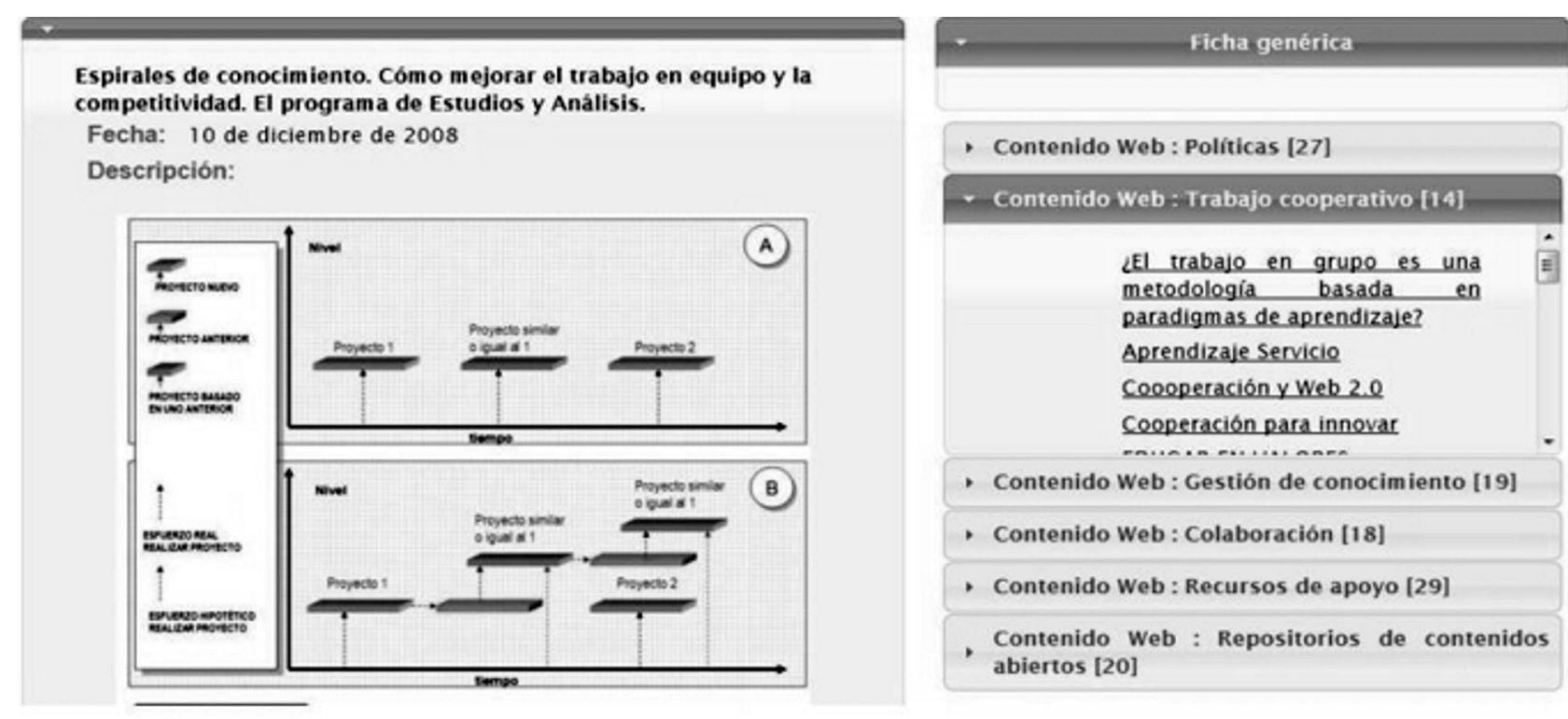

RELACIÓN CON LA REFERENCIA ENCONTRADA (I) EN EL METODO CSORA, PARA CADA REFERENCIA ENCONTRADA, SEMUESTRA OTRAS REFERENCIAS RELACIONADAS A TRAVES DEETIQUETAS. LASETIQUETAS ESTÁN AGRUPADAS EN UNA CATEG ORIA QUESEDENOMINA DERELACIÓN. EN LA FIGURA HA EN CONTRADO INFORMACIÓN RELACIONADA CON LAS SIGUIENTESETIQUETAS: (TRABAJO COOPERATIVO, GESTIÓN DEL CONOCIMIENTO, COLABORACIÓN, RECURSOS DEAPOYO YREPOSITORIOS DE CONTENIDOS EN ABIERTO).

ESTO SIGNFICA QUELA REFERENCIA ENCONTRADA TIENERELACION TANTO CON LAS ETIQUETASMOSTRADAS COMO CON LAS REFERENCIAS AGRUPADASEN CADAETIOUETA.

Figura 9. Relación de referencias agrupadas en etiquetas con un único elemento de búsqueda.

cualquier software 2.0, motores de búsqueda basados en la búsqueda por contenidos.

En los buscadores de internet también existe una tendencia (aunque muy tímida) a incorporar búsquedas basadas en etiquetas, y algunos de ellos integran las dos búsquedas por etiquetas y contenidos al mismo tiempo.

En el apartado anterior se ha expuesto el método CSO$R A$ para la búsqueda por etiquetas (características de las aplicaciones Web 2.0), sin embargo también integra la búsqueda por textos (característica de los motores de búsqueda por internet).

En la Figura 11 se muestra una búsqueda combinando etiquetas de búsqueda con contenidos. En este caso la búsqueda sería: "mostrar todas las referencias sobre web 2.0 que se utilicen en metodologías de evaluación o de colaboración y esta información la quiero para reflexionar.
Además en su contenido tiene que tener el texto "innovación educativa".

La ventaja técnica es que se pueden integrar las dos búsquedas (no hacer una u otra como en las actuales aplicaciones Web 2.0). Se permiten búsquedas por contenidos (adecuadas para cuando se sabe qué buscar) con etiquetas (adecuadas para cuando se sabe para qué se quiere utilizar la información). Esto permite obtener una mayor precisión de búsqueda y tener más posibilidades de que las referencias encontradas sean relevantes, ya que tienen la ventaja de integrar una búsqueda sabiendo qué se está buscando, para qué, dónde o por quién se quiere utilizar esa información.

La ventaja metodológica es la gran flexibilidad de las búsquedas y la orientación del buscador que permite eficacia cuándo se sabe perfectamente qué buscar, o cuando no se sabe qué buscar. Evidentemente la combi-

ARBOR Vol. 187 Extra 3 diciembre [2011] 51-66 ISSN: 0210-1963 


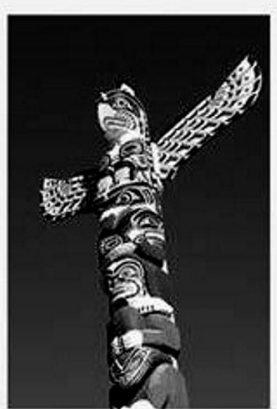

El titulo pretende coger la estela de la última frase del post de Elena de Benito

Quienes hemos decidido avanzar por el camino de la innovación educativa nos tropezamos continuamente con grandes piedras. Cualquier caminante que abre camino sabe que el camino no será fácil; pero tambièn sabe que al final tendrá dos recompensas:

Que logrará su meta

Que facilita el camino a otros que le sigan

En el camino de la innovación educativa, las recompensas no están tan claras ya que muchos caminantes no llegan a la meta (más bien abandonan antes) y que los que siguen ese camino se tropiezan una $y$ otra vez con las mismas piedras

Es posible que todos los que caminamos por esa senda, siempre nos hayamos preocupado de avanzar y no de quitar las piedras.

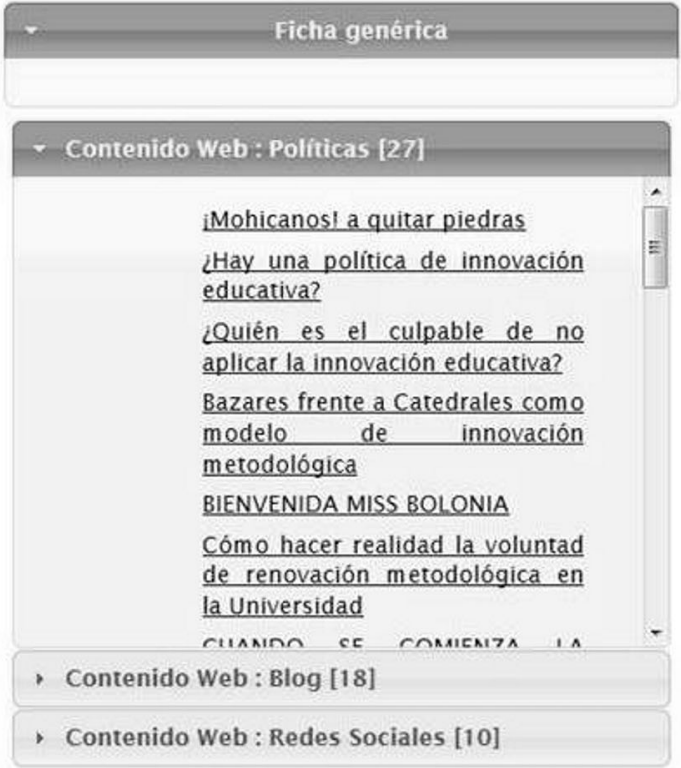

RELACIÓN CON LA REFERENCIA ENCONTRADA (II). LASETIQUETAS DE RELACIÓN NO SON LAS MISMAS, CAMBIAN EN FUNCIÓN DELA REFERENCIA ENCONTRADA, EN ESTE CASO LA INFORMACION DELA IZQUIERDA ESTÁRELACIONADA CON LASETIQUETAS (POLITICAS, BLOG Y REDES SOCIALES) Y EN CADAETIQUETARELACIONADA SEMUESTRAN MÁS REFERENCIAS.

Figura 10. Relación de referencias agrupadas en etiquetas con otro elemento del resultado de la búsqueda.

nación de ambas características permite una alta precisión de búsqueda.

\section{CONCLUSIONeS}

El método CSORA es un conjunto de enfoques metodológicos que permiten una mayor eficacia en los resultados de la búsqueda. Básicamente lo que se consigue es más adaptabilidad en las búsquedas según las necesidades de los usuarios, permitiendo realizar búsquedas tanto cuando se sabe que buscar como cuando no se sabe lo que se quiere, pero si se tiene claro para qué se quiere, o el contexto donde se va a utilizar la información.

Presenta un alcance mayor que el resto de buscadores, ya que dispone de un conjunto de metodologías para la clasificación, búsqueda, organización de resultados de la búsqueda e información relacionada con cada información encontrada.

Los buscadores clásicos presentan un algoritmo de búsqueda concreto y propio; los usuarios se deben adaptar a ese algoritmo. En el método CSORA se pueden definir los criterios de búsqueda, organización y relación, Por tanto, presenta un alto grado de adaptabilidad ya que es el usuario el que define "el algoritmo" de búsqueda.

Por otra parte, es un método muy adecuado para discriminar información en repositorios en los que haya una gran cantidad de información variada, así como para repositorios especializados con información muy similar.

El método CSORA se basa en innovaciones tecnológicas en gestión de etiquetas, ampliando la finalidad de las mismas y permitiendo la gestión de más de una etiqueta para las 


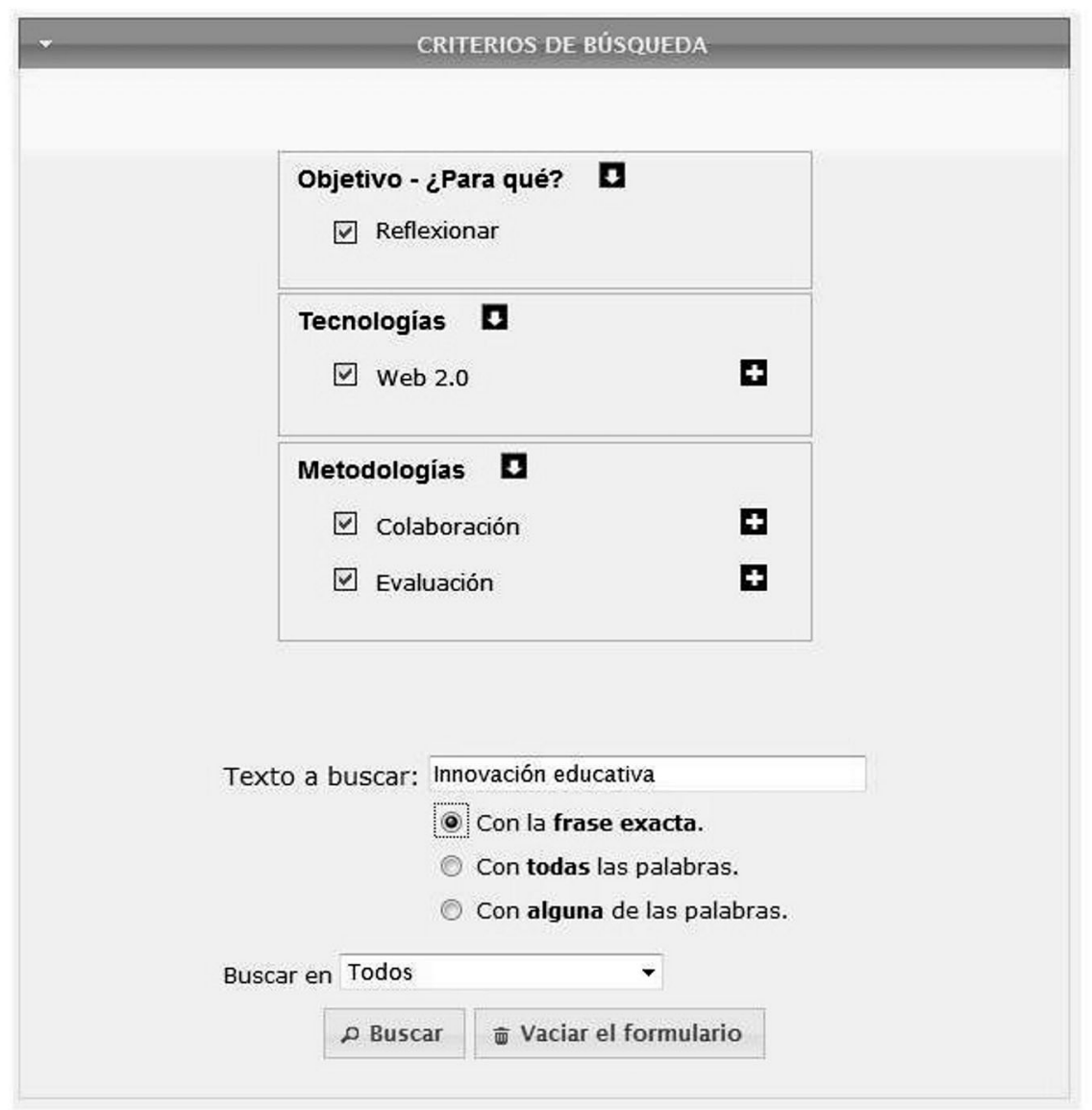

\begin{abstract}
MÉTODO DE BÚSQUEDA INTEGRADO. CSORA INTEGRA EL MÉTODO DE BÚSQUEDA POR TEXTOS CON EL DEETIQUETAS. EN EL EEMPLO LA BÚSQUEDA SERIAA: BUSCARINFORMACIÓN QUE:((OBJETIVO:Reflexionar.Y. TECNOLOGIAS:Web2.0.Y. METODOLOGÍAS(Colaboración. O. Evaluación)).Y. (CONTENIDO DELA REFERENCIA = "innovación Educativa")
\end{abstract}

Figura 11. Método de búsqueda integrado CSORA

búsquedas de información. Así mismo, integra las ventajas de distintos motores y modos de búsqueda (buscadores por etiquetas y contenidos).

En el LITI se ha desarrollado un buscador basado enteramente en las innovaciones tecnológicas y metodológicas del método CSORA. Dicho buscador (denominado Buscador 2.0 por ontologías) está siendo utilizado por diversas instituciones públicas (CDTI, Subdirección General de formación de profesorado e Innovación docente del Ministerio de Educación, ...) relacionadas con la innovación (tanto tecnológica como educativa) lo que prueba sobradamente su eficacia.
Además, se han realizado proyectos utilizando esta tecnología, como se muestra en Fidalgo, Balbín, Lerís y SeinEchaluce (2011).

Actualmente se están preparando nuevas características del método CSORA así como nuevas innovaciones tecnológicas relacionadas con la organización de la información. Todas las innovaciones metodológicas y tecnológicas se están implementando en la nueva versión del buscador (la 3.0) que estará operativa en 2012.

Por otra parte, otro campo inmenso de aplicación del buscador basado en el método CSORA es el del diseño de cur-

ARBOR Vol. 187 Extra 3 diciembre [2011] 51-66 ISSN: 0210-1963 
sos para el aprendizaje personalizado. La amplia variedad de información obtenida como resultado de las búsquedas combinando distintas etiquetas y categorías, facilitará el diseño instruccional de cursos adaptados a diferentes características de los usuarios. Estamos trabajando en esta línea, como se muestra en Sein-Echaluce, Lerís y Fidalgo (2011).

En las páginas http://innovacioneducativa.wordpress.com/ y http://www.gestionaconocimiento.com/ se muestran los prototipos $A$ y $B$ respectivamente.

\section{Agradecimientos}

Este trabajo ha sido posible gracias a los proyectos Munired y Mineral 2.0 (Programa Avanza. Ministerio de Industria, Turismo y Comercio). Especialmente relevante ha sido la contribución de José Carlos Baca, director del equipo de desarrollo y de Oscar Castañeda (LITI), que nos ha permitido utilizar datos del estudio que está realizando sobre buscadores.

\section{BIBLIOGRAFÍA}

Chakrabarti, S. (1999): Hypersearching the Web. Departament of Computer Sciencie. Cornell University. http:// www.cs.cornell.edu/home/kleinber/ sciam99.html (Último acceso el 29 de noviembre de 2011).

Fidalgo, A.; Balbin, A.; Leris, D. \& SeinEchaluce, M. L. (2011): Repositorio de mejores prácticas aplicadas en la educación superior en ingeniería. Promotion and Innovation with New Technologies in Engineering Education (FINTDI). Digital Object Identifier: 10.1109/FINTDI.2011.5945969 Publication Year: 2011, Page(s): 1 - 7. IEEE Conferences.

Gresman, K. (1988): Surfing with a purpose: Process and strategy put to the test on internet. Educom Review, 22-29.
Sein-Echaluce, M. L.; Fidalgo, A. y Gil, J. J. (2004): DSED: A New Technological Platform for E-learning, Collaborative Work and Knowledge Management. ED MEDIA 2004 World Conference on Educational Multimedia, Hypermedia \& Telecommunications. Lugano (Suiza). ISBN 1-880094-53-3. http:// www.editlib.org/?fuseaction=Reader. PrintAbstract\&tpaper_id=12692 (Último acceso el 29 de noviembre de 2011).

Sein-Echaluce, M. L.; Lerís, D. \&t Fidalgo, A. (2011): Diseño instruccional adaptativo de cursos online en Ingeniería. Promotion and Innovation with New Technologies in Engineering Education (FINTDI). Digital Object Identifier: $10.1109 /$ FINTDI.2011.5945972 Publication Year: 2011, Page(s): 1 - 8. IEEE Conferences. 